\title{
Service name resolution in Service-oriented network based on Kademlia algorithm Ziyan XU ${ }^{1, a}$, Jianya $\mathrm{CHEN}^{1, b}$
}

${ }^{1}$ Beijing University of Posts and Telecommunications, China

axzyan_0609@bupt.edu.cn, bjychen@bupt.edu.cn

Keywords: service-oriented network, name-resolving system, DHT network

\begin{abstract}
Today's Internet remains its original design that dates back more than three decades. In spite of tremendous heterogeneous networks and large scale of mobility devices, it still provides a single, basic, unreliable service offering. While this legacy architecture seemed so hard to support the requirements of new services and applications which are demanded by growing sophisticated, increasing rate of network flow and large-scale deployment, such as instant message, video on demand, electronic commerce, etc. In this paper, we describe a new identifier resolution KSR-NSN, which is based on SOFIA, a new service-oriented network architecture, that enables the diversity, quality, and huge amount of services.
\end{abstract}

\section{Introduction}

Over the last three decades, the Internet has been providing a ubiquitous communication infrastructure. As the core of the seven-layer protocol, IP layer act as the case when Internet first deployed, it only supports the basic communication paradigm: best-effort unicast packet delivery between two fixed end interfaces. While this original design is very hard to support current services, the tension arises from the fact that today's services are deployed all over the world and need dynamic changes; too many users create a large amount of network flow, best-effort unicast paradigm puts extreme pressure to our backbone network; with the surge of mobile devices, IP address is insufficient to supply all the devices. To face the challenges above, research on the future network architecture can be divided into to directions. Some researchers want to resolve these problems through "patching” the existing network architecture, this Incremental method still uses TCP/IP protocol as the thin waist of network protocol stack, which cannot solve the mobility, scalability, controllability problems from within. Another part of researchers intend to change current network architecture from scratch, in spite of the constraints of existing architecture. This kind of clean-state thinking has gradually become the consensus of the future network architecture design.

To resolve the backbone network traffic surging, the NSF (National Science Foundation) started a project named FIND. NDN[1] as a subproject of FIND, uses content as the central part of network, which aims at decoupling the content provider with physical host, content request will no further rely on the specific content provider address. NDN has been confirmed to have good performance in date-intensive applications, but for interaction-intensive applications, NDN needs to periodically broadcast probe packages, which resulting in a large amount of signaling overhead.

As a part of NSF's another subproject FIA, MobilityFirst[2] resulted in a new clean-state mobility-centric architecture, it uses a self-certifying public key network addresses to support strong authentication and security, separates naming from addressing via a global dynamic name resolution 
service.

SOFIA[3], which is brought up by ICT (Institute of Computing Technology, Chinese Academy of Science), replaces IP with a global unified service ID which is the code of a specific service, another label "locator" is utilized to specify the physical location of service provider, thus realizing the separation of service from location. Communication in SOFIA is driven by services[4], thus the Internet will focus on service request and responses; it naturally accommodates mobility, the roaming mobile devices only changes their access points but not their services; a new global SID will also resolve the scalability problem.

\section{The Sketch of SOFIA}

SOFIA (Service-oriented Future Internet Architecture) uses Service ID as its thin waist of protocol stack, like the figure below:

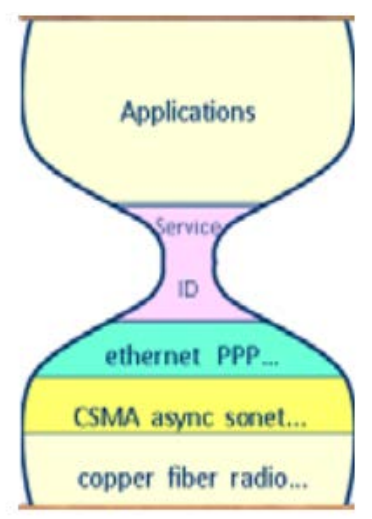

Fig. 1. SOFIA Protocol Stack

Communication in SOFIA is driven by services. There are three kinds of basic service operations in SOFIA including Service Registration, Service Updating and Service Request. Once a user wants to get a service, a service request will be sent to a SOFIA router, thus this request will be delivered to the optimal service provider. Service Registration has the function for service provider to publish a new service, which is similar with Service Update procedure. They all have the purpose to register a service provider's location to others.

With SOFIA network architecture, how to organize SIDs and the way to resolve it has become an open project, KSR-NSN (Kademlia-based Service Routing on Named Service Network) is one of the solutions to cope with the large-scale network in SOFIA.

\section{Layered Service Name Resolution}

According to the difference of capability in network routers, KSR-NSN divides identifier resolve procedure into two layers, namely service layer on the top and routing layer on the bottom. Routers in service layer, which is called CSR (Core Service Router), have more computing power and storage capacity to provider more complicated routing policy and the management to ESR (End Service Router) in routing layer. For each CSR, all the ESRs belong to it with itself can be regarded as an AS (Autonomous Region), service request will be routed in the AS first to find a matched service. 


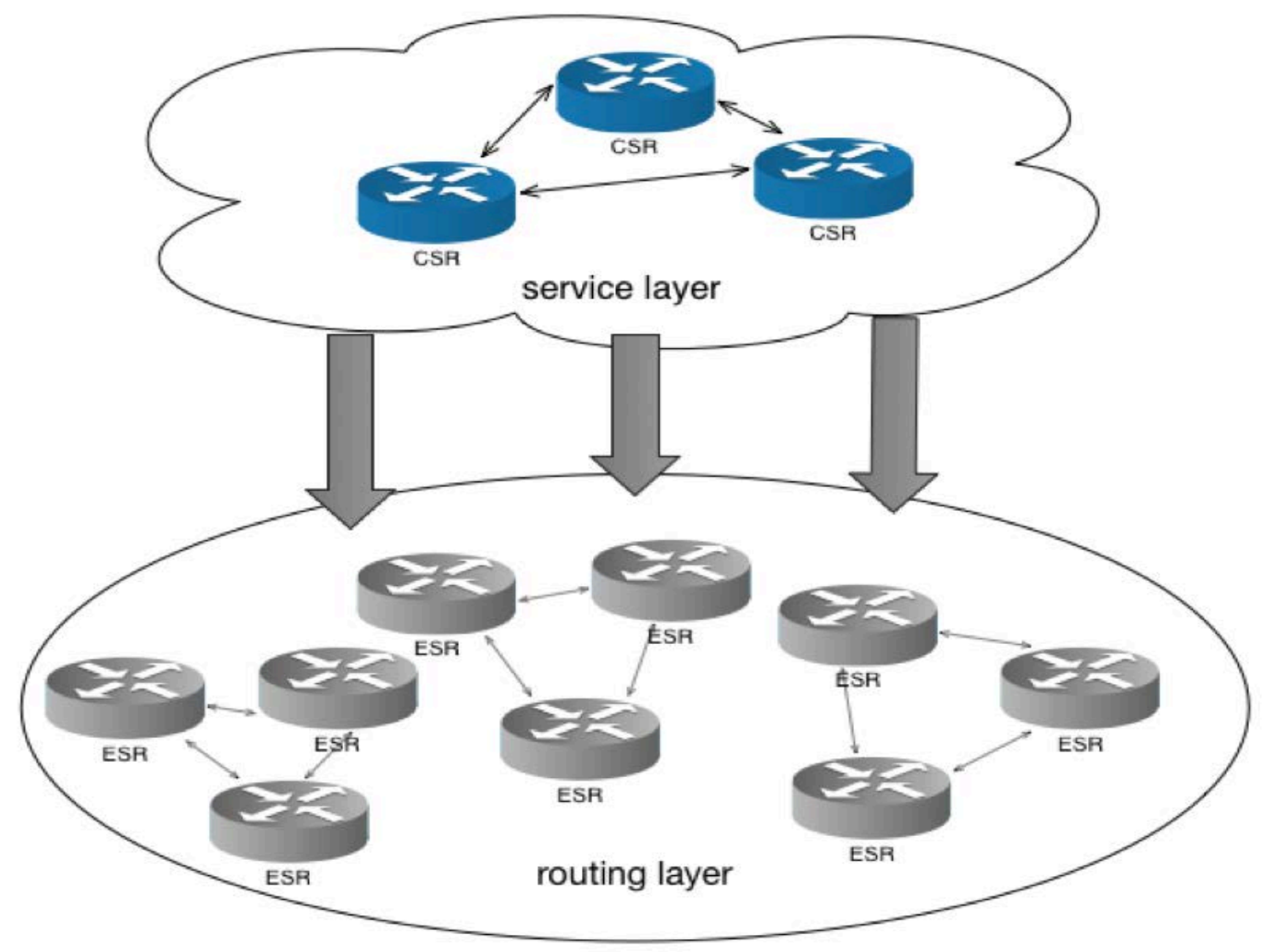

Fig. 2. KSR-NSN Layered Architecture

Each SID should be normalized to one paradigm, that the service request can be acknowledged by the Internet. Similar to NDN's name structure, we use a hierarchical name structure to describe a specific service.

\begin{tabular}{|c|c|c|}
\hline Prefix & Name & Significance \\
\hline bupt.edu.cn & Highest name prefix & The scope of a specific service \\
\hline Video & Service type & Type of service \\
\hline fnl.mov & Service name & Specific name of service \\
\hline V $<$ timestamp $>$ & Version & Version of service \\
\hline $60<$ FPS $>$ & Frame & The meta information of service \\
\hline
\end{tabular}

Table 1. Service Name Structure

As is shown in Table 1, the combination of every prefix can completely represent the whole content of a service, ESRs would take the maximum matching algorithm to route at the routing layer. In service layer, we provide another identifier to locate a service that is located outside the AS, called SID', SID' can be calculated as (1).

$$
S I D^{\prime}=\operatorname{hash}(S I D)
$$

SID' will be routed through AS using Kademlia[5] algorithm, which means each CSR has an only 160-bit ID as its node ID, called NID. For every service operation, ESR would route SID in the local AS, if fail, CSR will generate an SID' to locate this service through AS. The KSR-NSN is worked as follows: 
Service Request. When a user wants to get a video stream service, say $S I D=$ bupt.edu.cn/Video/fni.mov/V<20150908 $>/ 60<$ FPS $>$, ESR in access network will route this SID through local AS, if it failed to fetch any satisfied service in local AS, SID' will be generated according to (1), CSR of the AS will deliver this SID' to suitable CSRs to fetch where the service should be located, then the remote CSR will re-route SID through its AS to find the specific location of the service and send it back.

Service Registration. When a service provider published a service named $S I D=$ bupt.edu.cn/Paper/SOFIA.pdf/V<20130517>/high $<$ Quality $>$, ESR will take this SID and store it with its actual service provider in local AS, then CSR of the AS would generate SID' to route in service layer to find other AS to store information of the service, chosen CSRs then send SID through AS to add items in ESR's routing table and register the service in the AS.

Service Update. Service update is similar to the operation of service registration, but the former one replace the register process with update.

\section{Evaluation and Simulation}

Assume that routers contain all the routing information in network, the uncertainty of route will be diminished. If there are $d_{i}$ network interfaces in each router, each packet forwarding to any interface has the same probability $\mathrm{p}_{\mathrm{i}}$,

$$
p_{i}=1 / d_{i}
$$

For each router, the entropy $C_{i}$ of it can be calculated as (3).

$$
C_{i}=-\sum_{i=1}^{d_{i}}\left(p_{i} * \log _{2} p_{i}\right)=\log _{2} d_{i}
$$

Since we know the entropy of each router, the total entropy for a network that contains $n$ routers can be calculated as below.

$$
C=\sum_{i=1}^{n} C_{i}=n \log _{2} d_{i}
$$

As shown in (4), with the growth of routers, the entropy of network will be increasing in a linear trend. If the routers can't be able to cover all the routing information in the whole network, or update the information immediately, the routing performance will be decreased.

With KSR-NSN, when a service need to be distributed, local AS can preferentially router the information through the routing layer, obtaining physical distance advantage when routing with SID. While routing extraterritorial, SID' get highly routing polymerization because of its flat name structure, can provide crossing path to different AS. By weighing the size of each AS, KSR-NSN can be well effective to overcome scalability problem in the internet, control routing information entropy, provide a fast, stable and service-oriented name resolution approach in SOFIA. 


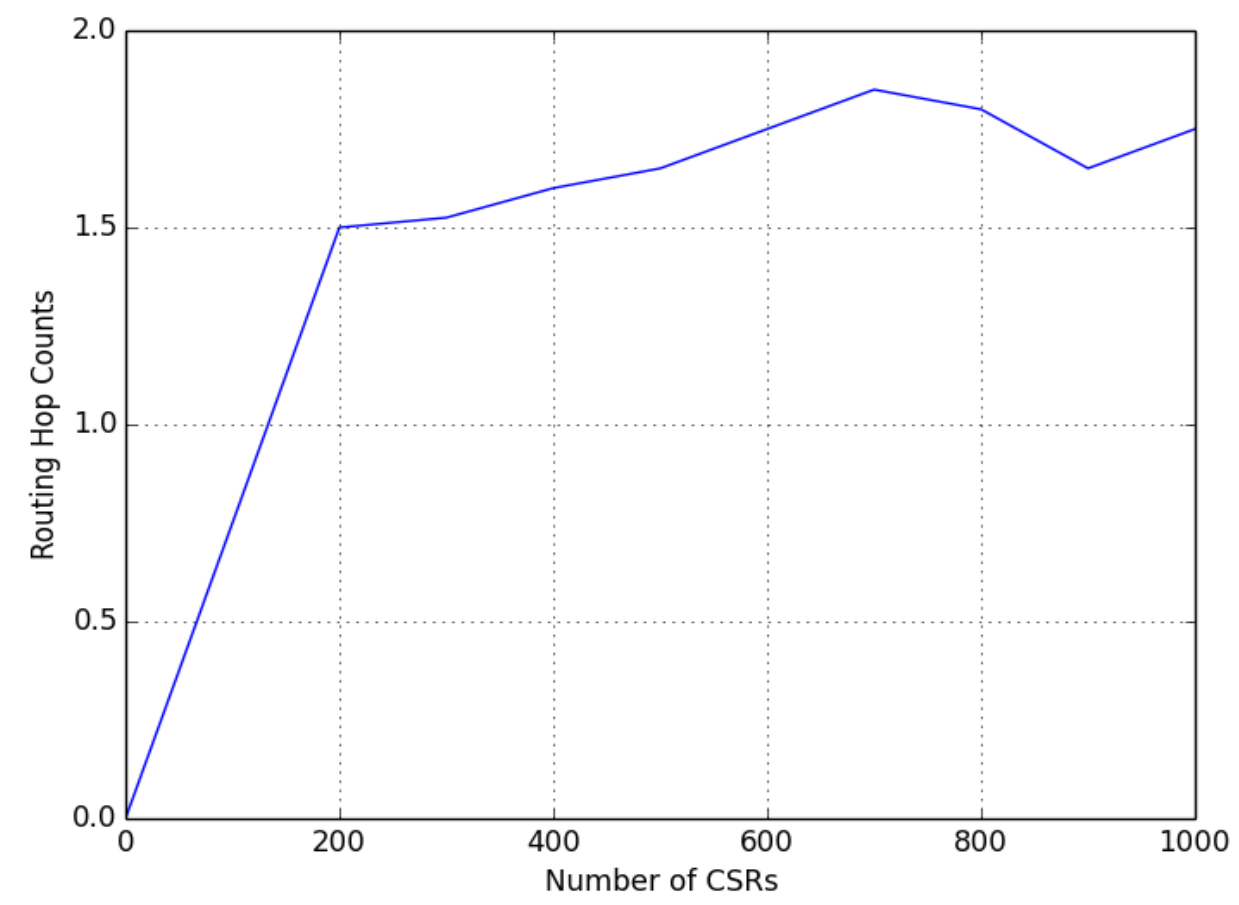

Fig. 3. Relationship between routing hop counts and number of CSRs

Fig. 3 shows the increasing trend in routing hop counts is more flat than a linear straight line while the numbers of CSRs keep rising. Using Kademlia algorithm, its k-bucket data-structure and first-out elimination strategy make KSR-NSN act well in service information routing through service layer with the keep growth of the network scale.

Comparing with NDN strategy, assuming the $50 \%$ of the service request should be resolved outside the local AS, KSR-NSN still has its goodness in routing performance, as shown in Fig. 4.

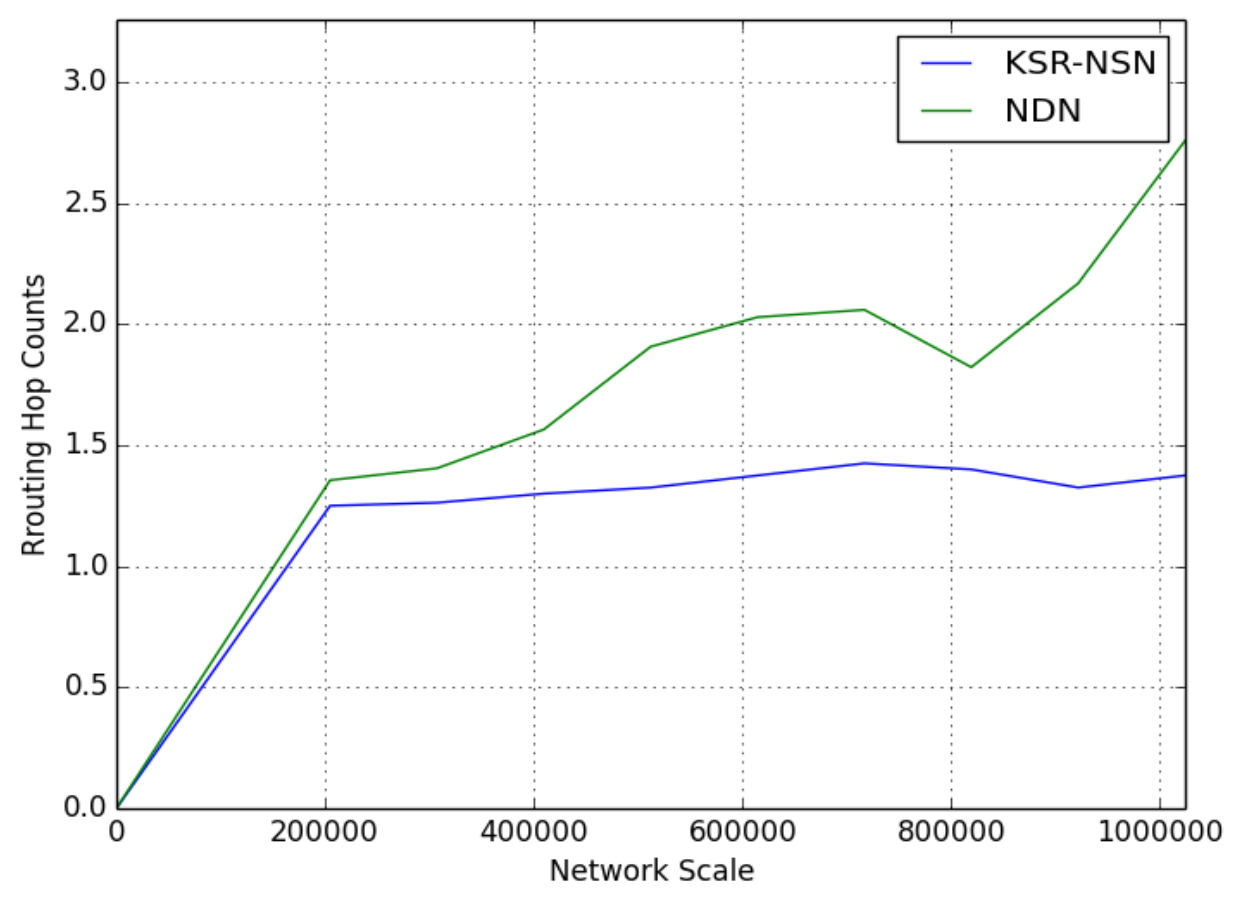

Fig. 4. Relationship between routing hop counts and network scale 


\section{Summary}

In this paper, we highlighted the inadequacies of current Internet design to satisfy the requirements of emerging services and a clean-state service oriented network architecture SOFIA. Based on SOFIA, we described a two-layer service name resolving architecture KSR-NSN. In service layer, we use Kademlia protocol to route through different autonomous regions, which allows requirements such as availability, robustness, mobility and scalability. In routing layer, a hierarchical name structure is applied to route in local autonomous region to make the resolve process fast and QoS sensitive. At last, we build a simulation program to verify the performance of KSR-NSN in large-scale network.

\section{References}

[1] Zhang L, Estrin D, Burke J, et al. Named data networking (ndn) project[J]. Relatório Técnico NDN-0001, Xerox Palo Alto Research Center-PARC, 2010.

[2] MobilityFirst Future Internet Architecture Project, http://mobilityfirst.winlab.rutgers.edu/.

[3] Xie G, Sun Y, Zhang Y, et al. Demo Abstract: Service-Oriented Future Internet Architecture (SOFIA)[J]. IEEE Infocom/Poster, Shanghai, China, 2011.

[4] Erl T. Service-oriented architecture: concepts, technology, and design[M]. Pearson Education India, 2005.

[5] Maymounkov P, Mazieres D. Kademlia: A peer-to-peer information system based on the xor metric[M]//Peer-to-Peer Systems. Springer Berlin Heidelberg, 2002: 53-65. 